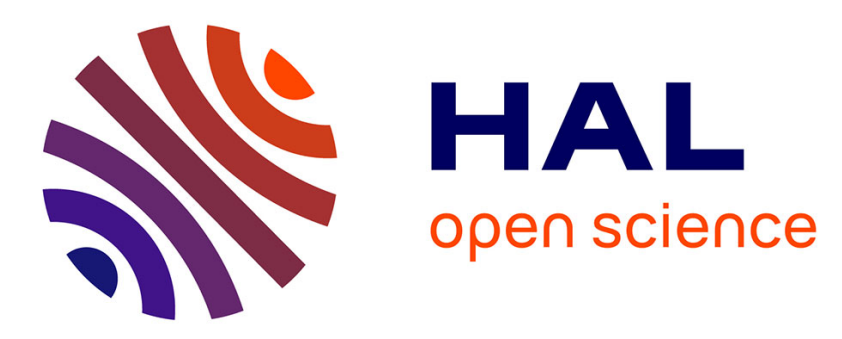

\title{
Modeling and 1D Control of a non Contact Magnetic Actuation Platform at the Air/Liquid Interface for Micrometer Scale Applications
}

Mohamed Dkhil, Aude Bolopion, Stéphane Régnier, Michaël Gauthier

\section{- To cite this version:}

Mohamed Dkhil, Aude Bolopion, Stéphane Régnier, Michaël Gauthier. Modeling and 1D Control of a non Contact Magnetic Actuation Platform at the Air/Liquid Interface for Micrometer Scale Applications. International Conference on Manipulation, Automation and Robotics at Small Scales, Jul 2016, Paris, France. hal-02868205

\section{HAL Id: hal-02868205 https://hal.science/hal-02868205}

Submitted on 15 Jun 2020

HAL is a multi-disciplinary open access archive for the deposit and dissemination of scientific research documents, whether they are published or not. The documents may come from teaching and research institutions in France or abroad, or from public or private research centers.
L'archive ouverte pluridisciplinaire HAL, est destinée au dépôt et à la diffusion de documents scientifiques de niveau recherche, publiés ou non, émanant des établissements d'enseignement et de recherche français ou étrangers, des laboratoires publics ou privés. 


\title{
Modeling and 1D Control of a non Contact Magnetic Actuation Platform at the Air/Liquid Interface for Micrometer Scale Applications
}

\author{
Mohamed Dkhil ${ }^{1,2}$, Aude Bolopion ${ }^{1}$, Stéphane Régnier ${ }^{2}$ and Michaël Gauthier ${ }^{1}$
}

\begin{abstract}
Non contact magnetic actuation inside liquids or in ambient environments has gained a large interest over the last few years. A large range of micrometer size particles are controlled using this principle. However little consideration has been made to air/liquid interface, despite the interesting properties of this environment (good repeatability due to the small adhesion forces, and low viscous effects that enable to reach large velocities). This work aims at providing models to perform both the conception and the control of these new devices. Both an accurate analytical model for the conception, and a simplified one that can be easily invertible in real time for the control are proposed. Different assumptions based on the small scale of the particle are discussed to obtain the simplified model. The validity of the approach is analysed using a 1D trajectory control experiment based on the simplified model of the system. This work provides the basic understanding for the future developments of magnetic actuation systems at the air/liquid interface for micrometer scale applications.
\end{abstract}

\section{INTRODUCTION}

Magnetic actuation is used to control robots for a wide range of applications, such as minimally invasive medical procedures [1], cells manipulation [2], [3] and parallel assembly at the microscale [4]. They are based on the use of an artificial magnetic structure called microrobot that can be powered and controlled by a magnetic source [1], [5]. The size of the microrobots ranges from a few micrometers to a few millimeters.

Magnetic energy is a promising solution to actuate objects with high speed [5], [6], [7], [8]. A wide range of microrobots are controlled using several coils or electromagnets [9], [10], [11], [12]. To give only a few examples, helical microrobots are moved using a rotating magnetic field [13], [14]; some bacteria such as Tetrahymena pyriformis are actuated by magnetic field and used as microrobots [15], [16], [17]. This success can be attributed to the ability of magnetic actuation to produce fast displacements. The systems presented above are either in ambient environments or in liquids. However at micrometer scale adhesion forces are a major issue in ambient environments, and the repeatability of the control is not guaranteed [18]. In liquid medium the velocity is limited due to the viscous forces.

The approach proposed in this work is to consider a different environment, the air/liquid interface, to benefit from both the good repeatability due to small adhesion and the

\footnotetext{
1 Institut FEMTO-ST, UBFC, UFC, ENSMM, UTBM, CNRS UMR 6174, 24 rue Alain Savary, 25000 Besançon, France. mohamed.dkhildfemto-st.fr, aude.bolopionefemto-st. fr

${ }^{2}$ Institut des Systèmes Intelligents et de Robotique, Université Pierre et Marie Curie, CNRS UMR 7222, 4 Place Jussieu, 75005 Paris, France. regnierdisir.upmc.fr
}

the high velocity due to the low viscous forces. Only very few works report studies on interfaces [19], [20]. To develop magnetic actuation systems on this new environment there is a need of models to perform both the conception of the device and the control of the trajectory of the particles. This study fulfils two goals: to provide accurate models for the conception, and easily invertible models for real time closed loop control. The basic forces applied on the particle are thus presented. A generic analytical model of a magnetic actuation system at the air/liquid interface is then provided. To answer the two objectives of this paper different assumptions are made, considering the small scale of the particles. Corresponding models are presented, that can be used for control. The validity of the approach is verified by performing 1D closed loop control based on one of the simplified model proposed.

This paper is organized as follows. Sec. II presents the forces applied on the particle by a magnetic actuation system at the air/liquid interface. The generic model, as well as simplified ones are proposed in Sec. III. The approach is validated by $1 \mathrm{D}$ closed loop control using one of the simplified model in Sec. IV. The last section concludes the paper.

\section{Magnetic ACtuation System AT ThE AIR LiQuid INTERFACE}

Four main forces are applied to the particle: (i) the magnetic force $\vec{F}_{m}$ induced by the coils, (ii) the weight of the particle $\vec{P}$, (ii) the force resulting from the surface tension $\vec{T}$ and (iv) the drag force $\vec{F}_{d}$ (Fig. 1). The expression of each force should be obtained to present the dynamic model of the movement of the particle at the air/liquid interface. This is the goal of this section.

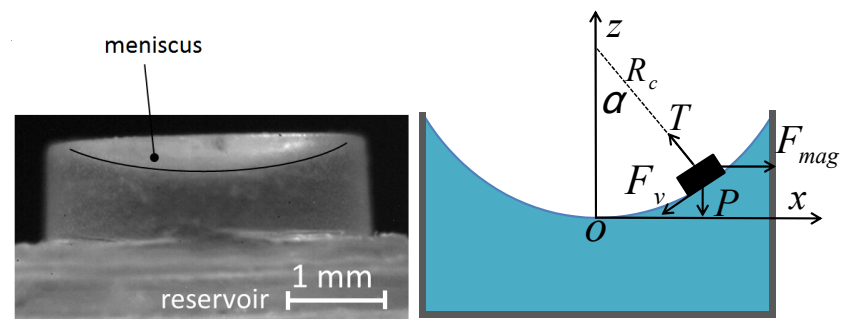

Fig. 1. Non contact microrobotic at the air liquid/interface. The left picture depicts a side view of the reservoir on which is placed the magnetic microparticle. The meniscus can be clearly seen. The right picture details the forces applied to the particle at an equilibrium position (the drag force, which depends on the velocity, is not depicted) 


\section{A. Magnetic force}

The magnetic force applied to a magnetic particle is expressed by:

$$
\vec{F}_{m}=V(\vec{M} \cdot \vec{\nabla}) \vec{B}
$$

where $\vec{M}=\chi \vec{B}$ is the magnetization of the particle, $\chi$ is the magnetic susceptibility of the particle and $\vec{B}$ is the magnetic field. The magnetic field is produced by several coils. For an ideal soft magnetic material with negligible hysteresis and if the system is operated with the cores of the coils in their linear magnetization domain it can be considered that the total magnetic field is the sum of the magnetic field produced by each coil [21]. The identification of the contribution of a single coil is thus sufficient.

The magnetic field produced by one coil can be approximated by a single function, named $f$ in this work, under the assumption that the axial magnetic field depends only on the position along the axis of the coil (it is constant for any radial position). This hypothesis holds for any radial positions situated next to the axis of the coil. In this work, for the considered workspace (see Fig. 2) the variation of the axial magnetic field for a radial position $r=0$ and for a radial position $r=2 \mathrm{~mm}$ is less than $15 \%$. In that case:

$$
B_{1 x}=f(x) I_{1}, \quad B_{1 y}=-\frac{y}{2} f^{\prime}(x) I_{1}, \quad B_{1 z}=-\frac{z}{2} f^{\prime}(x) I_{1} .
$$

In the case of four coils that are orthogonal (see Fig. 2), the magnetic force is given by:

$$
\left(\begin{array}{c}
F_{m x} \\
F_{m y} \\
F_{m z}
\end{array}\right)=V \chi\left(\begin{array}{c}
2 f_{i}(x) f_{i}^{\prime}(x) I_{i}^{2}+f_{j}^{\prime}(y) f_{i}(x) I_{i} I_{j} \\
2 f_{j}(y) f_{j}^{\prime}(y) I_{j}^{2}-f_{i}^{\prime}(x) f_{j}(y) I_{i} I_{j} \\
0
\end{array}\right)
$$

where $i=1$ or 3 and $j=2$ or $4 . f_{i}$ and $f_{j}$ refer to:

$$
\left\{\begin{array}{l}
f_{i}(x)=\cos \left(\frac{\pi(i-1)}{2}\right) f\left(x \cos \left(\frac{\pi(i-1)}{2}\right)\right) \\
f_{j}(y)=\sin \left(\frac{\pi(j-1)}{2}\right) f\left(y \sin \left(\frac{\pi(j-1)}{2}\right)\right)
\end{array}\right.
$$

where $B_{1 x}=f(x) I_{1}, B_{2 y}=f(y) I_{2}, B_{3 x}=-f(-x) I_{3}$ and $B_{4 y}=$ $-f(-y) I_{4}$.

Each coil is powered depending on the sign of the desired currents $I_{x}$ and $I_{y}$. If $I_{x}>0($ resp. $<0)$ coil 1 (resp. 3) is powered and $I_{1}=I_{x}\left(\right.$ resp. $\left.I_{3}=-I_{x}\right)$. If $I_{y}>0($ resp. $<0)$ coil 2 (resp. 4) is powered and $I_{2}=I_{y}$ (resp. $I_{4}=-I_{y}$ ). As an example, if coils 1 and 2 are powered the magnetic force is:

$$
\left\{\begin{array}{l}
F_{m x}=V \chi\left(2 f(x) f^{\prime}(x) I_{x}^{2}+f^{\prime}(y) f(x) I_{x} I_{y}\right) \\
F_{m y}=V \chi\left(2 f(y) f^{\prime}(y) I_{y}^{2}-f^{\prime}(x) f(y) I_{x} I_{y}\right)
\end{array}\right.
$$

\section{B. Surface tension}

The micro-object is placed at the air/liquid interface. Since the workspace is small (up to some millimeters), a meniscus appears on the reservoir, which must be taken into account while modeling the motion (see Fig. 1). The weight of the micro-object $\vec{P}$ is compensated by the resultant force of the surface tension $\vec{T}$. The equilibrium condition is given by:

$$
\gamma C \sin (\phi)=m g
$$

where $\gamma$ is the surface tension, $C$ is the perimeter of the object, $\phi$ is the contact angle between the micro-object and the liquid, $m$ is the mass of the object and $g=9.8 \mathrm{~m} . \mathrm{s}^{-2}$ is the gravitational acceleration. To evaluate the deformation of the meniscus due to the presence of the object the $\phi$ angle is evaluated numerically. A rectangular nickel object is considered (density: $\rho=8902 \mathrm{~kg} / \mathrm{m}^{3}$, size: $100 \times 90 \times 25 \mu \mathrm{m}^{3}$ ) and the liquid is water (surface tension: $\gamma=7210^{-3} \mathrm{~N} / \mathrm{m}$ ). Based on Eq. (6) the $\phi$ angle is about $7.210^{-4} \mathrm{rad}$ which is negligible. The shape of the meniscus is not affected by the weight of the micro-object with the above mentioned properties.

\section{Drag force}

The force applied by the surrounding fluids on the object includes the viscous force applied by the air on the object and the shear force due to the contact between the object and the liquid surface. The viscous force applied by the air is negligible compared to the shear force, due to the difference between the air and the liquid viscosity. The shear force depends on the viscosity of the fluid, the object geometry and the contact angle between the object and the liquid surface [22]. Considering the Stokes law it is expressed by:

$$
\vec{F}_{v}=-k \vec{v}
$$

where $k$ is the Stockes coefficient and $\vec{v}$ is the velocity of the object. $k$ depends on the geometry of the object, the viscosity of the liquid, and the contact between the particle and the air/liquid interface. In case of a spherical particle $k=6 \pi r \eta f_{s}$ where $r$ is the particle radius and $f_{s}$ is a dimensionless coefficient. Its value depends on the contact between the particle and the liquid surface. In this study the geometry of the particles is more complex since they are parallelepipeds. The numerical value of $k$ will thus be identified experimentally, as explained in Sec. IV-B.

\section{MOdEL OF THE SYSTEM}

\section{A. Generic analytical model}

Based on Newton's second law, the movement of the particle can be written:

$$
m \vec{a}=\vec{P}+\vec{F}_{m}+\vec{F}_{d}+\vec{T}
$$

where $\vec{a}$ and $m$ are respectively the acceleration and the mass of the particle. This equation can be written along the three 
axes $(o, \vec{x}),(o, \vec{y})$ and $(o, \vec{z})$ :

$$
\left\{\begin{array}{c}
m \ddot{x}=F_{m x}-k v \cos (\alpha) \cos (\theta)-T \sin (\alpha) \cos (\theta) \\
m \ddot{y}=F_{m y}-k v \cos (\alpha) \sin (\theta)-T \sin (\alpha) \sin (\theta) \\
m \ddot{z}=F_{m z}-P-k v \sin (\alpha)+T \cos (\alpha) \\
z=\zeta(x, y) \\
\tan (\alpha)=\frac{d \zeta(x, y)}{d\left(\sqrt{x^{2}+y^{2}}\right)}, \tan (\theta)=\frac{d y}{d x}
\end{array}\right.
$$

where $\alpha$ is the angle between the vertical axis $(o, \vec{z})$ and the axis normal to the upper side of the particle, $\theta$ is the angle between the $(o, \vec{x})$ axis and the straight line drawn from the origin $o$ and the projection of the position of the particle in the $(x, y)$ plane and $\zeta(x, y)$ is the function that defines the $z$ coordinates of the particle as it moves in the $(x, y)$ plane. Since the particle moves on the surface of the meniscus the following relation holds:

$$
T=\left(-F_{m z}+P\right) \cos (\alpha)+\left(F_{m x} \cos (\theta)+F_{m y} \sin (\theta)\right) \sin (\alpha)
$$

The position of the particle can be written as: $x=$ $R_{c} \sin (\alpha) \cos (\theta), \quad y=R_{c} \sin (\alpha) \sin (\theta)$ and $z=R_{c}(1-$ $\cos (\alpha)$ ), where $R_{c}$ is the radius of curvature of the meniscus. Based on these equations and on Eq. (9) and Eq. (10) the generic model of the movement of the particle can be written as:

$$
\left\{\begin{array}{c}
m \ddot{x}+k \dot{x}+\frac{x}{R_{c}}\left(1-\frac{z}{R_{c}}\right)\left(m g-F_{m z}\right)= \\
\left(1-\left(\frac{x}{R_{c}}\right)^{2}\right) F_{m x}-\frac{x y}{R_{c}^{2}} F_{m y} \\
m \ddot{y}+k \dot{y}+\frac{y}{R_{c}}\left(1-\frac{z}{R_{c}}\right)\left(m g-F_{m z}\right)= \\
\left(1-\left(\frac{y}{R_{c}}\right)^{2}\right) F_{m y}-\frac{x y}{R_{c}^{2}} F_{m x} \\
m \ddot{z}+k \dot{z}+\left(\frac{2 z}{R_{c}}-\left(\frac{z}{R_{c}}\right)^{2}\right)\left(m g-F_{m z}\right)= \\
\frac{x}{R_{c}}\left(1-\frac{z}{R_{c}}\right) F_{m x}+\frac{y}{R_{c}}\left(1-\frac{z}{R_{c}}\right) F_{m y}
\end{array}\right.
$$

These equations represent the generic model of a non contact magnetic actuation system at the air/liquid interface. It can be used for the conception of the future devices. However it is non linear and not easily invertible for real time control of the system. Next sections will thus discuss some assumptions to simplify this model.

\section{B. Hypothesis of small movements, large radius of curvature of the meniscus}

In this work the particle displacements are considered as small compared to the radius of curvature of the meniscus. This assumption leads to: $x<<R_{c}, y<<R_{c}$ and $z<<R_{c}$ where $x, y$ et $z$ are the displacements on each of the three axes respectively.

For example, in this work the radius of curvature of the meniscus is equal to $10.5 \mathrm{~mm}$ and the displacements along $x$ and $y$ are around $300 \mu \mathrm{m}$. The vertical displacement, due to the meniscus, is around $3 \mu \mathrm{m}$. This is 100 times less than along $x$ and $y$ axes. The study will thus be limited to the displacements in the $(x, y)$ plane. The first order terms, $x / R_{c}$ and $y / R_{c}$, are 20 times larger than the second order terms, $\left(x / R_{c}\right)^{2},\left(y / R_{c}\right)^{2}, x y / R_{c}^{2}, x z / R_{c}^{2}$ and $y z / R_{c}^{2}$. The generic model (Eq. (11)) can then be simplified:

$$
m \ddot{x}+k \dot{x}+\left(\frac{m g}{R_{c}}\right) x=F_{m x}, \quad m \ddot{y}+k \dot{y}+\left(\frac{m g}{R_{c}}\right) y=F_{m y}
$$

C. Hypothesis of small movements, small variation of the magnetic gradient

The hypothesis of small movements around the center of the meniscus can also have a consequence on the expression of the magnetic force. In that case the gradient of the magnetic field can be considered as constant, and its value will be the one at point $(0,0)$. From Eq. (3) and (4) it can be seen that:

$$
\left\{\begin{array}{l}
F_{m x}^{F}=V \chi f(0) f^{\prime}(0) \operatorname{sign}\left(I_{x}\right)\left(2 I_{x}^{2}+I_{x} I_{y}\right) \\
F_{m y}^{F}=V \chi f(0) f^{\prime}(0) \operatorname{sign}\left(I_{y}\right)\left(2 I_{y}^{2}-I_{x} I_{y}\right)
\end{array}\right.
$$

D. Hypothesis of negligible radial component of the magnetic field

The expression of the magnetic force can also be simplified by considering that the radial component of the magnetic field can be neglected. In that case the magnetic force becomes:

$$
\left\{\begin{array}{l}
F_{m x}^{F R}=2 V \chi f(0) f^{\prime}(0) \operatorname{sign}\left(I_{x}\right) I_{x}^{2} \\
F_{m y}^{F R}=2 V \chi f(0) f^{\prime}(0) \operatorname{sign}\left(I_{y}\right) I_{y}^{2}
\end{array}\right.
$$

\section{E. Hypothesis of neligeable inertia}

As the size of the objects decreases the inertia can be neglected. The goal of this paragraph is to define the value of this critical size for the proposed application. This analysis is performed for spherical objects, for which an analytical expression of the viscous force is available. The movement of the magnetic particle is performed along the axis of one of the coil, chosen to be the $(o, x)$ axis. The hypothesis of small movements is considered as valid. Based on Eq. (12), the dynamic model of the movement of the particle is:

$$
m \ddot{x}+k \dot{x}+\left(\frac{m g}{R_{c}}\right) x=F_{m x}
$$

The $k$ coefficient is computed as explained in Section IV-B. The magnetic force is computed as explained in Eq. (14). It is considered that coil 1 is powered, so $I_{x}=I_{1}$ and $I_{1}>0$. Based on these expressions and the dynamic model (Eq. 15) the movement of the particle can be written as:

$$
m \ddot{x}+6 \pi r \eta f_{s} \dot{x}+\left(\frac{m g}{R_{c}}\right) x=2 V \chi f(0) f^{\prime}(0) I_{1}^{2}
$$

Using the change of variable $\tilde{I}_{1}=I_{1}^{2}$ and the Laplace transform, the transfer function of the system is:

$$
\frac{X(s)}{\tilde{I}_{1}(s)}=\frac{2 V \chi f(0) f^{\prime}(0)}{m s^{2}+6 \pi r \eta f_{s} s+\frac{m g}{R_{c}}}
$$


Inertia can be neglected if the poles of the denominator of the transfer function are distant. The two poles are equal when the discriminant of the equation $m s^{2}+6 \pi r \eta f_{s} s+\frac{m g}{R_{c}}$ is null, which implies:

$$
\left(6 \pi r \eta f_{s}\right)^{2}-4 \frac{m^{2} g}{R_{c}}=0
$$

By substituting the mass of the particle by its expression based on the volume of the particle (radius $r$ ) and its bulk density $\rho_{p}$ a critical radius $r_{l}$ is obtained:

$$
r_{l}=\left[\frac{81 R_{c}}{16 g}\left(\frac{\eta f_{s}}{\rho_{p}}\right)^{2}\right]^{\frac{1}{4}}
$$

For particles with a radius lower than the critical radius $r_{l}$, the characteristic equation has two real solutions. The system is damped, and can be approximated by a first order system where inertia is neglected. The numerical values of the coefficient $f_{s}$ are between 0.54 and 0.68 [22]. The corresponding critical radius is comprised between $66 \mu \mathrm{m}$ and $75 \mu \mathrm{m}$ for a spherical particle.

In this work the particles are not spherical but parallelepiped, with a maximum size of $100 \times 90 \times 25 \mu^{3}$. The volume, and thus the inertia are equivalent to a sphere with a radius lower than $38 \mu \mathrm{m}$. In addition, the surface friction of parallelepipeds is larger than the one of spheres, which increase the contribution of viscous effects. The critical radius of a sphere with large viscous effects will be larger than the one obtained in this study (see Eq. 19). Since the radius of a sphere equivalent to the particles used in this work is lower than the critical value (between $66 \mu \mathrm{m}$ and $75 \mu \mathrm{m}$ ), and since the effect of viscous forces is underestimated, inertia can be neglected here. The dynamic model can then be written as:

$$
k \dot{x}+\left(\frac{m g}{R_{c}}\right) x=F_{m x}, \quad k \dot{y}+\left(\frac{m g}{R_{c}}\right) y=F_{m y}
$$

\section{F. Synthesis of the models}

The previous sections present several simplifications of the generic model (Eq. 11). Table I summarizes the corresponding models. The following notations are used:

- $D$ stands for the hypothesis of small displacements (the radius of curvature of the surface of the meniscus is considered as large),

- I denotes that the inertia is neglected,

- $F$ indicates that due to the hypothesis of small displacements the magnetic field gradient can be considered as constant,

- $R$ means that the radial component of the magnetic field is neglected.

These models fulfil the twofold goal of a precise model for the conception of the system (using $M_{\text {gene }}$ ), and a model that can be invertible in real time for the control (using $M_{\mathscr{H}_{D I F R}}$ for example).

\section{1D TRAJECTORY CONTROL}

In this section the simplified model is tested for 1D trajectory control.

\section{A. Setup}

The setup used in this work is presented in Fig. 2. Nickel micro-objects with a rectangular shape are fabricated using micro-fabrication technologies. One of these micro-objects is placed on an air/water interface (distilled water is used). Acquisition of images is performed by a "Photonfocus" camera mounted on a Mitutoyo M plan Apo 5X objective. The reservoir used is $4 \mathrm{~mm}$ of diameter. Only the central part of the reservoir is recorded by the camera. The images are imported to a computer using a PCI interface. The PC runs software on a real time Unix Kernel. The software is written in $\mathrm{C}$ using OpenCV libraries for image processing. It controls the signals sent to the coils via a National instrument card (NI-PCI-6733). The coils are powered by an electronic amplifier (ST TL048CN) which amplifies the control signals issued from the NI card with a response time equal to $0.1 \mu \mathrm{s}$. Details can be found in [20].

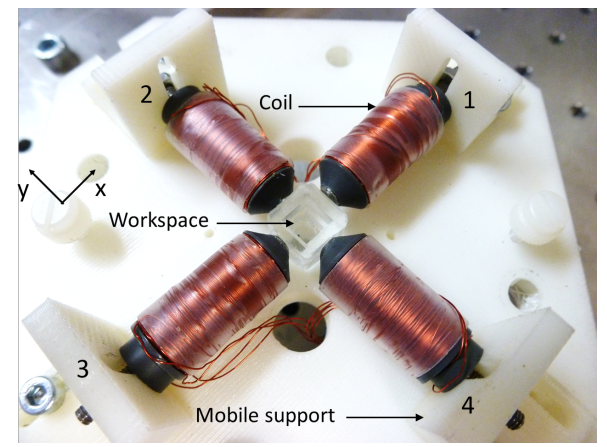

Fig. 2. Setup of the magnetic actuation system at the air/liquid interface

\section{B. Identification of the model}

The trajectory control of the particle is based on the simplified model $M_{\mathscr{H}_{D I F R}}$ developed in Sec. III. A basic control law is implemented to test the performances of the system. The numerical values of the parameters of the model, and in particular of the viscous coefficient $k$ and the magnetic susceptibility $\chi$ must first be identified. Since the value of these parameters vary greatly depending on the fabrication process of the particles it is difficult to find accurate values in the literature for the particles used in this work. An identification method based on experimental data is presented in [20]. The values given in Tab. II are used in the following.

The function $f$ introduced in Sec. II-A in Eq. (2) is identified experimentally by performing magnetic field measurements using a teslameter. A five order polynomial function is fitted: $f(x)=\sum_{i=0}^{5} a_{i} x^{i}$ where $a_{0}=0.02, a_{1}=5.5, a_{2}=826$, $a_{3}=1.3710^{5}, a_{4}=4.5610^{7}$ and $a_{5}=610^{9}$. 
TABLE I

SUMMARY OF THE MODELS DEVELOPED.

\begin{tabular}{|c|c|}
\hline Model & Equations \\
\hline$M_{\text {gene }}$ & $\begin{array}{c}m \ddot{x}+k \dot{x}+\frac{x}{R_{c}}\left(1-\frac{z}{R_{c}}\right)\left(m g-F_{m z}\right)=\left(1-\left(\frac{x}{R_{c}}\right)^{2}\right) F_{m x}-\frac{x y}{R_{c}^{2}} F_{m y}, \\
m \ddot{y}+k \dot{y}+\frac{y}{R_{c}}\left(1-\frac{z}{R_{c}}\right)\left(m g-F_{m z}\right)=\left(1-\left(\frac{y}{R_{c}}\right)^{2}\right) F_{m y}-\frac{x y}{R_{c}^{2}} F_{m x}, \\
m \ddot{z}+k \dot{z}+\left(\frac{2 z}{R_{c}}-\left(\frac{z}{R_{c}}\right)^{2}\right)\left(m g-F_{m z}\right)=\frac{x}{R_{c}}\left(1-\frac{z}{R_{c}}\right) F_{m x}+\frac{y}{R_{c}}\left(1-\frac{z}{R_{c}}\right) F_{m y},\end{array}$ \\
\hline$M_{\mathscr{H}_{D}}$ & $m \ddot{x}+k \dot{x}+\left(\frac{m g}{R_{c}}\right) x=F_{m x}$, \\
\hline$M_{\mathscr{H}_{D I}}$ & $k \dot{x}+\left(\frac{m g}{R_{c}}\right) x=F_{m x}, \quad k \dot{y}+\left(\frac{m g}{R_{c}}\right) y=F_{m y}$ \\
\hline$M_{\mathscr{H}_{D I F}}$ & $k \dot{x}+\left(\frac{m g}{R_{c}}\right) x=F_{m x}^{F}$ \\
\hline$M_{\mathscr{H}_{D I F R}}$ & $k \dot{y}+\left(\frac{m g}{R_{c}}\right) y=F_{m y}^{F R}$ \\
\hline
\end{tabular}

TABLE II

NUMERICAL VALUES OF THE DYNAMIC MODEL

\begin{tabular}{|l|c|r|}
\hline Parameter & Symbol & Value \\
\hline density of the particle & $\rho_{p}$ & $8902 \mathrm{~kg} / \mathrm{m}^{3}$ \\
\hline magnetic susceptibility & $\chi$ & $1.510^{3} \mathrm{kA} /(\mathrm{m} . \mathrm{T})$ \\
\hline viscous coefficient & $k$ & $3.3910^{-7} \mathrm{~kg} \cdot \mathrm{s}^{-1}$ \\
\hline radius of curvature of the meniscus & $R_{c}$ & $10.5 \mathrm{~mm}$ \\
\hline dynamic viscosity of the liquid & $\eta$ & $10^{-3} \mathrm{~Pa} . \mathrm{s}$ \\
\hline density of the liquid & $\rho$ & $1000 \mathrm{~kg} / \mathrm{m}^{3}$ \\
\hline gravitational acceleration & $g$ & $9.8 \mathrm{~m} / \mathrm{s}^{2}$ \\
\hline
\end{tabular}

\section{1D trajectory control}

A control law is implemented to control the trajectory of a small object along the $(o, x)$ axis along a sinusoidal trajectory (Fig. 3). This control law is based on the use of a proportional derivative controller on the first bloc of control to improve the rapidity. The motion is ensured by two coils located in the same axis opposite each other. Two experiments are performed on a $60 \times 50 \times 25 \mu^{3}$ object. Firstly, the proposed control law has been tested on low speed trajectory, a sinusoidal trajectory with $500 \mathrm{~ms}$ period and a magnitude of $100 \mu \mathrm{m}$ around the workspace center (Fig. 4(a)). The error between the experimental trajectory and the reference is less than $7 \mu \mathrm{m}$ which represents $4 \%$ of the micro-object size and the maximum velocity achieved is about $1.25 \mathrm{~mm} / \mathrm{s}$.

Secondly, the control law proposed is tested for a high speed trajectory, a sinusoidal trajectory 5 times faster (Fig. 4(b)). As seen in the figure, the micro-object starts its motion after a delay of $6 \mathrm{~ms}$. This is due to the fact that it first rotates around its center of mass to align its magnetization vector with the magnetic field. The same rotation occurs each time the direction of motion changes. The same phenomena does appear in Fig. 4(a) but can hardly be seen due to the time scale of the figure. This

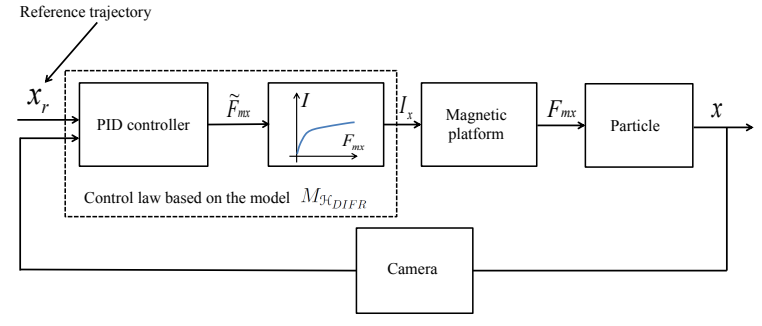

Fig. 3. Control scheme for closed loop 1D trajectory tracking

rotation can be avoided by powering the two coils with a current of opposite sign. At the time $t=6 \mathrm{~ms}$ the object starts to follow the reference trajectory. The position error is less than $10 \%$ of the object size and the maximum velocity achieved is about $6 \mathrm{~mm} / \mathrm{s}$.

These results demonstrate that closed loop control can be achieved using the developed simplified model.

\section{CONCLUSion}

This work presents the model of a non contact magnetic actuation system at the air/liquid interface. The aim is to provide a framework for the conception and control of these devices. Conception requires an accurate and generic analytical model, whereas control necessitates a numerical model that can be easily invertible in real time. This paper presents the generic model of these devices. Several assumptions are discussed, namely simplifications of the magnetic force based on linearisation and on the fact that the radial component of the magnetic field can be neglected, simplifications due to the small movements of the particle on the meniscus and simplifications due to the small inertia of the particle. Corresponding models are presented. A 1D closed loop control of sinusoidal trajectory is demonstrated using the $M_{\mathscr{C}_{D I F R}}$ model. Experiments show that a precise 


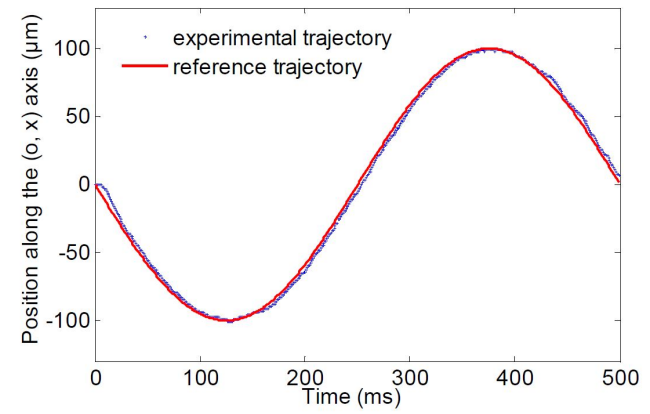

(a) Experimental trajectory of the micro-object while tracking a sinusoidal trajectory of $500 \mathrm{~ms}$ period. The proportional constant is equal to $710^{-6} \mathrm{mN}^{-1}$ and the derivative constant is equal to $57 \mathrm{~ms}$.

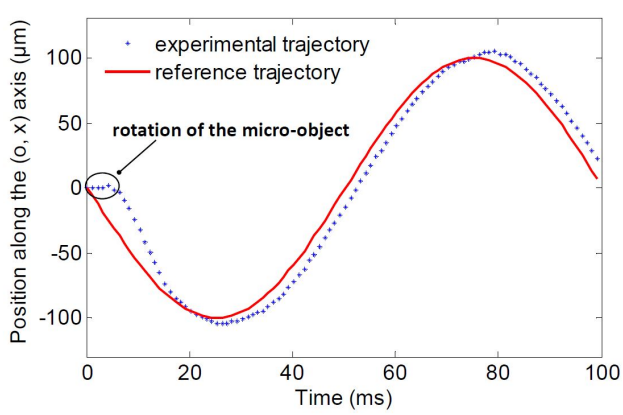

(b) Experimental trajectory of the micro-object while tracking a sinusoidal trajectory of $100 \mathrm{~ms}$ period. The proportional constant is equal to $1.210^{-5} \mathrm{mN}^{-1}$ and the derivative constant is equal to $72 \mathrm{~ms}$.

Fig. 4. Experimental closed loop trajectory tracking. These experiments are performed using a $60 \times 50 \times 25 \mu^{3}$ object. The camera acquisition period is $1 \mathrm{~ms}$. During the first six milliseconds the object rotate to align its magnetization and the magnetic field.

trajectory tracking is obtained for a $60 \times 50 \times 25 \mu \mathrm{m}^{3}$ particle for a trajectory of $500 \mathrm{~ms}$ period. High velocities $(6 \mathrm{~mm} / \mathrm{s})$ can be obtained but the precision is around $10 \%$ of the size of the particle.

This work is only the first step towards versatile 2D controlled non contact magnetic actuation system at the air/liquid interface. However it provides the basic understanding for both the conception and the control of the future devices. Future works include extending the control law to 2D trajectory tracking at high speed.

\section{ACKNOWLEDGMENT}

This work has been supported by the French Agence Nationale de la Recherche, through the LEMA project (contract "ANR 12 BS03 007 01"), by the Labex ACTION project (contract "ANR-11-LABX-01-01"), by the "Région Franche Comté" and by the French RENATECH network and its FEMTO-ST technological facility.

\section{REFERENCES}

[1] B. J. Nelson, I. K. Kaliakatsos, and J. J. Abbott, "Microrobots for minimally invasive medicine," Annual review of biomedical engineering, vol. 12 , pp. $55-85,2010$.
[2] H. Uvet, L. Feng, S. Ohashi, M. Hagiwara, T. Kawahara, Y. Yamanishi, and F. Arai, "On-chip single particle loading and dispensing," in IEEE International Conference on Robotics and Automation, 2011, pp. 3151-3156.

[3] N. Inomata, T. Mizunuma, Y. Yamanishi, S. Kudo, and F. Arai, "Onchip magnetically driven micro-robot for enucleation of oocyte," in International Symposium on Micro-NanoMechatronics and Human Science, 2009, pp. 493-498.

[4] B. R. Donald, C. G. Levey, and I. Paprotny, "Planar microassembly by parallel actuation of mems microrobots," Microelectromechanical Systems, Journal of, vol. 17, no. 4, pp. 789-808, 2008.

[5] C. Pawashe, S. Floyd, E. Diller, and M. Sitti, "Two-dimensional autonomous microparticle manipulation strategies for magnetic microrobots in fluidic environments," IEEE Transactions on Robotics, pp. 467-477, 2012.

[6] H. Marino, C. Bergeles, and B. J. Nelson, "Robust electromagnetic control of microrobots under force and localization uncertainties," IEEE Transactions on Automation Science and Engineering, vol. 11, no. 1 , pp. $310-316,2014$.

[7] Z. Ye and M. Sitti, "Dynamic trapping and two-dimensional transport of swimming microorganisms using a rotating magnetic microrobot," Lab Chip, vol. 14, pp. 2177-2182, 2014.

[8] S. Bouchebout, A. Bolopion, J.-O. Abrahamians, and S. Régnier, "An overview of multiple dof magnetic actuated micro-robots," Journal of Micro-Nano Mechatronics, vol. 7, no. 4, pp. 97-113, 2012.

[9] D. R. Frutiger, K. Vollmers, B. E. Kratochvil, and B. J. Nelson, "Small, fast, and under control: wireless resonant magnetic micro-agents," The International Journal of Robotics Research, vol. 29, no. 5, pp. 613636, 2010.

[10] E. B. Steager, M. S. Sakar, C. Magee, M. Kennedy, A. Cowley, and V. Kumar, "Automated biomanipulation of single cells using magnetic microrobots," The International Journal of Robotics Research, vol. 32 , no. 3, pp. 346-359, 2013.

[11] Z. Ye, E. Diller, and M. Sitti, "Micro-manipulation using rotational fluid flows induced by remote magnetic micro-manipulators," Journal of Applied Physics, vol. 112, no. 6, pp. 064 912-064 912, 2012.

[12] I. A. Ivan, G. Hwang, J. Agnus, M. Rakotondrabe, N. Chaillet, and S. Régnier, "First experiments on magpier: a planar wireless magnetic and piezoelectric microrobot," in IEEE International Conference on Robotics and Automation, 2011, pp. 102-108.

[13] K. E. Peyer, L. Zhang, and B. J. Nelson, "Bio-inspired magnetic swimming microrobots for biomedical applications," Nanoscale, vol. 5 , no. 4, pp. 1259-1272, 2013

[14] T. Xu, G. Hwang, N. Andreff, and S. Régnier, "Modeling and swimming property characterizations of scaled-up helical microswimmers," Transactions on Mechatronics, 2013.

[15] I. S. Khalil, M. P. Pichel, L. Zondervan, L. Abelmann, and S. Misra, "Characterization and control of biological microrobots," in Experimental Robotics. Springer, 2013, pp. 617-631.

[16] S. Martel, "Bacterial microsystems and microrobots," Biomedical microdevices, vol. 14, no. 6, pp. 1033-1045, 2012.

[17] D. H. Kim, P. S. S. Kim, A. A. Julius, and M. J. Kim, "Threedimensional control of engineered motile cellular microrobots," in IEEE International Conference on Robotics and Automation, 2012, pp. 721-726.

[18] S. Bouchebout, A. Bolopion, M. Kharboutly, I. A. Ivan, J. Agnus, and S. Régnier, "Design and first experiments on magpier, the magnetic microrobot," in IEEE International Symposium on Optomechatronic Technologies, 2012

[19] J. A. Piepmeier, S. Firebaugh, and C. S. Olsen, "Uncalibrated visual servo control of magnetically actuated microrobots in a fluid environment," Micromachines, vol. 5, no. 4, pp. 797-813, 2014.

[20] M. Dkhil, M. Kharboutly, A. Bolopion, S. Régnier, and M. Gauthier, "Closed-loop control of a magnetic particle at the air-liquid interface," Automation Science and Engineering, IEEE Transactions on, vol. PP, no. 99, pp. 1-13, 2015

[21] M. P. Kummer, J. J. Abbott, B. E. Kratochvil, R. Borer, A. Sengul, and B. J. Nelson, "Octomag: An electromagnetic system for 5-dof wireless micromanipulation," IEEE Transactions on Robotics, pp. 1006-1017, 2010.

[22] J. T. Petkov, N. D. Denkov, K. D. Danov, O. D. Velev, R. Aust, and F. Durst, "Measurement of the drag coefficient of spherical particles attached to fluid interfaces," Journal of colloid and interface science, vol. 172, no. 1, pp. 147-154, 1995. 